\title{
Low Reynolds Number Rotor Blade Aerodynamic Analysis
}

\author{
Tytus Tulwin ${ }^{1, *}$ \\ ${ }^{1}$ Lublin University of Technology, Department of Thermodynamics, Fluid Mechanics and Aircraft Propulsion, Nadbystrzycka 38D, \\ 20-618 Lublin, Poland
}

\begin{abstract}
Maintaining a steady hover flight in a rotorcraft usually requires high energy input. The aim of the paper is to prove that it is possible to vastly reduce energy use in a rotorcraft by reducing the disc loading. The energy consumption reduction is especially important in electric rotorcraft, where the energy source is characterized by low energy density when compared to the hydrocarbon fuel in ICE rotorcraft. The paper presents results of CFD simulations on low Reynolds Number operating rotors. For low RE rotors tip vortex induced drag is highly affecting the rotor's Figure of Merit, thus reducing rotor performance. Even though FM is reduced, the low RE setup is still beneficial in terms of reduced Power Loading, the main factor responsible for hover endurance.
\end{abstract}

\section{Introduction}

Over the years the rotor performance was being improved making rotorcraft a safe and competitive form of transportation. Modern helicopters fly at Figure of Merit around 0.8 at high Reynolds Numbers of $>1 \times 10^{6}$. Although increasing Reynolds Number is good for higher FM, the Power Loading which defines the power to lift ratio decreases making rotorcraft very power demanding. We can decrease PL by decreasing the Disk Loading, increasing the Figure of Merit or using the forward flight induced power benefits. One way of reducing the power required for flight is to increase forward velocity. By doing this the mass flow of air passing through the disk is increased while the induced velocity is not [1]. A good analysis of helicopter in forward flight can be found here [2]. In this paper [3] author developed a model for flapping flight if birds and insects - one scenario of low Reynolds Number hovering flight. Another model for low-RE flight conditions can be found here [4]. The highest low-RE figure of merit found in the literature was 0.67 at $\mathrm{RE}<30$ '000 [5] [6] [7] [8]. There are papers providing good airfoils for lowRE conditions [9]. A good model for induced power of helicopter flight can be found here [10]. Recent research showed some good results for low Reynolds number in co-axial configuration in low density conditions [11].There are papers analysing microrotors at even lower RE at microscales [12]. Wind turbines also can also experience low-RE conditions [13]. The aim of this paper is to find out the feasibility of using low-RE rotor conditions in hover flight in order to reduce the power needed by the rotorcraft. There is lack of papers analysing low-RE rotor conditions at larger rotor diameter scales $(>0.5 \mathrm{~m})$.

\section{Materials and Methods}

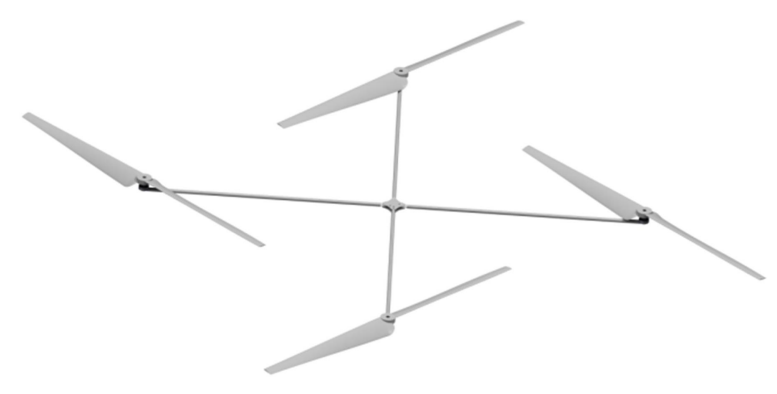

Fig. 1. Quadrocopter test rig CAD model.

In order to design a custom, efficient, low RE rotor three methods were used in order to account for the phenomena: momentum theory based mathematical model, blade element theory simulations and computational fluid dynamics simulations with ANSYS software. After many iterations first blade geometry propositions were established. Basic parameters to achieve are shown in table 1 . The average Reynolds Number on the blade is set to 150 '000 resulting from the lowered Disk Loading parameter. The Reynolds Number reduction is inevitable as the rotor rotational speed decreases with increased diameter. Figure 1 shows the proposed qudrocopter test rig geometrical model with low $\mathrm{RE}$ rotors.

\footnotetext{
${ }^{*}$ Corresponding author: t.tulwin@pollub.pl
} 


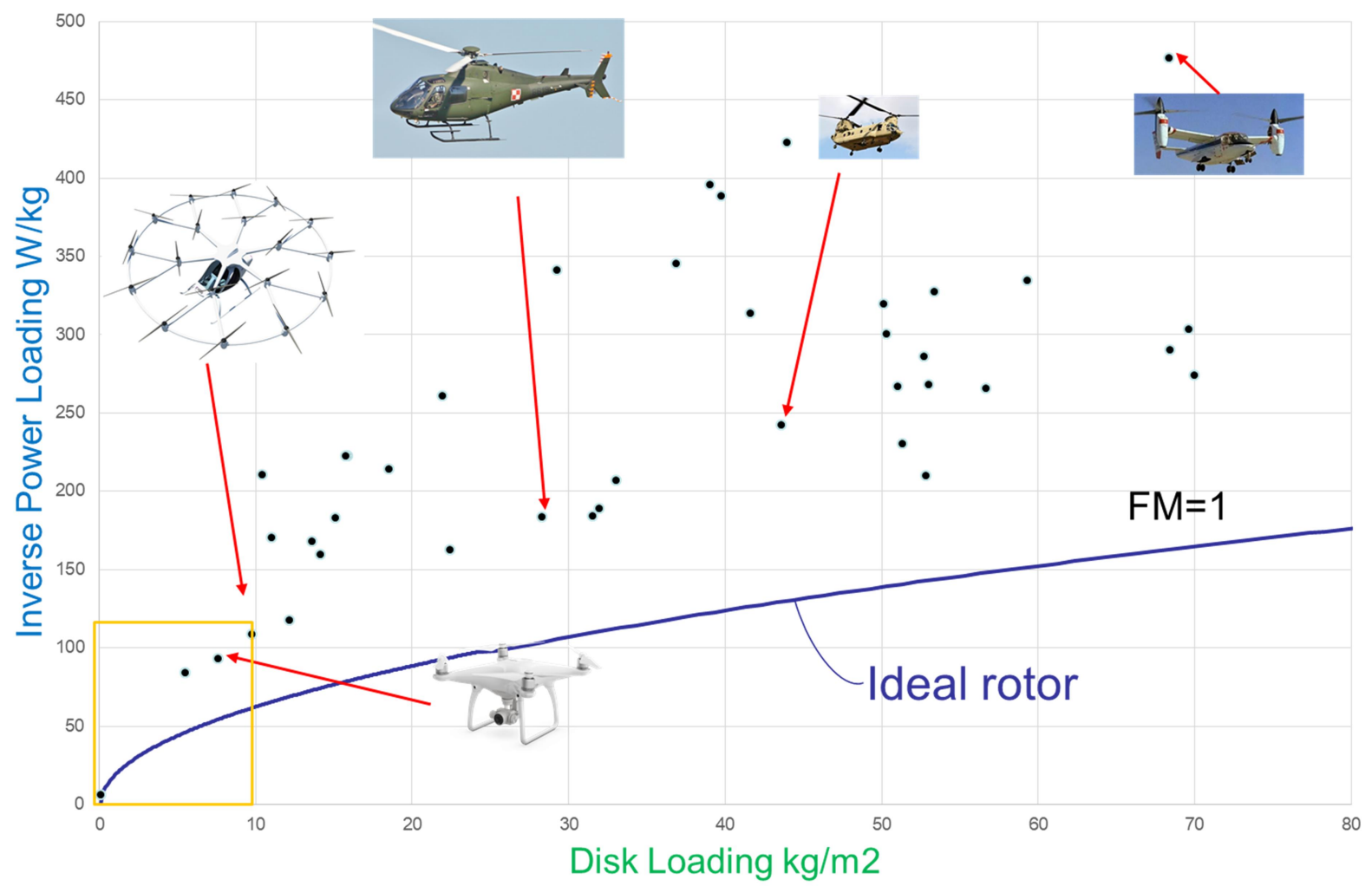

Fig. 2. The power loading characteristics for different disk loadings, with different rotorcraft hover performance data plotted.

The turbulence model used in the simulation was kepsilon with SST and Standard Wall Function boundary layer treatment. In order to decrease the computational time and still maintain small mesh error adaptive refinement was used. The simulation method was a Frozen Rotor Steady State. The rotation is defined as rotating reference frame allowing for accurate wake vortices without going to transient simulation.

Fig 2 show inverse power loading characteristics in respect to the disk loading with real rotorcraft hover performance data points plotted. It is clear that for high Reynolds Number rotors the maximum achievable Figure of Merit is around 0.8. The rectangle shows the desired area of interest for low energy usage, further presented on fig. 3 .

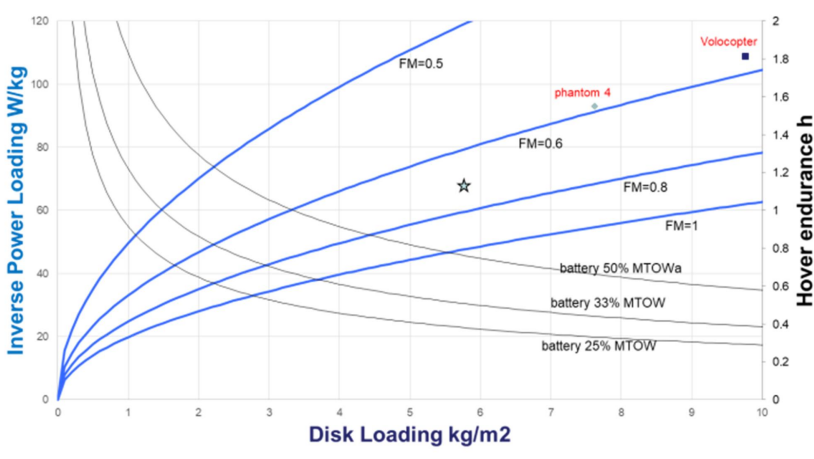

Fig. 3. The power loading characteristics and hover endurance for different disk loadings, with Figure of Merit iso-lines.
JBlade software was used for the Blade Element Theory (BET) analysis. BET method is useful for first design concept simulations. The CFD software used for 3 dimensional analysis is Ansys Fluent. K-w SST turbulence model was used with adaptive mesh based on vorticity iso value criterion.

Fig. 3 show inverse power loading characteristics in respect to the disk loading, at different constant values of FM (left scale). Also hover endurance is shown assuming for different battery weight ratios (right scale). At proposed disk loading of $6 \mathrm{~kg} / \mathrm{m} 2$ hover endurance of 0.8 hours is achievable.

\section{Results and Discussion}

Results prove the feasibility of low Reynolds Number at low Disk Loading as plotted on Fig. 3. The results from theoretical model, BET analysis and CFD analysis prove that using low RE rotor blades is achievable. Figure 4 presents the iso-contour profiles of constant vorticity with turbulence kinetic energy map color data. The vortices at the tip of the blades are clearly visible and have a strong effect on the Figure of Merit decrease. Another reason for decreasing FEM is the less turbulent boundary layer separation region that is beneficial for lift coefficient. At Disk Loading $5.76 \mathrm{~kg} / \mathrm{m} 2$ the inverse power loading value of $68 \mathrm{~W} / \mathrm{kg}$ was achieved allowing for around 50 minute hover endurance assuming that $50 \%$ of rotorcraft weight is a high density Li-ion battery (Fig 3.). The vorticity map plotted on the rotor domain side-view cross-section shows the vortex sheet distribution along the blade. 


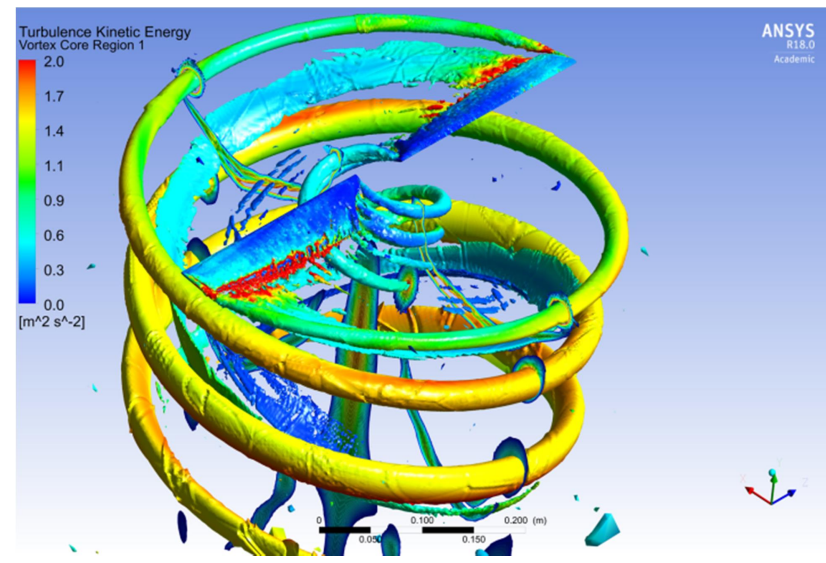

Fig. 4. Vorticity iso-contour with turbulence kinetic energy color map.

The vorticity parameter was a main criterion for simulation adaptive refinement. Vorticity defines the highest viscosity induced flow gradients very well making the velocity flow map detailed (Fig. 6).

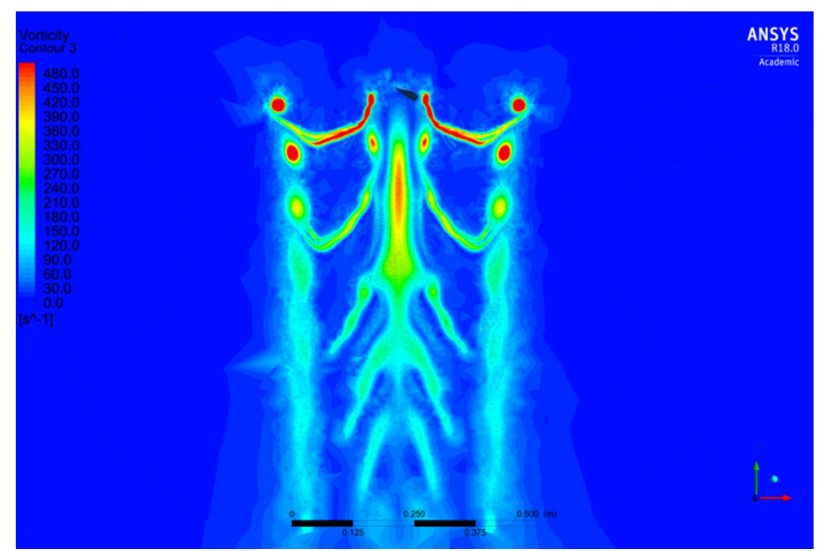

Fig. 5. Vorticity map for z-axis plane view.

The velocity map follows the Fraude Momentum Theory [14] where the flow velocity is highest at some distance below the rotor area after the increased pressure is exchanged to kinetic energy.

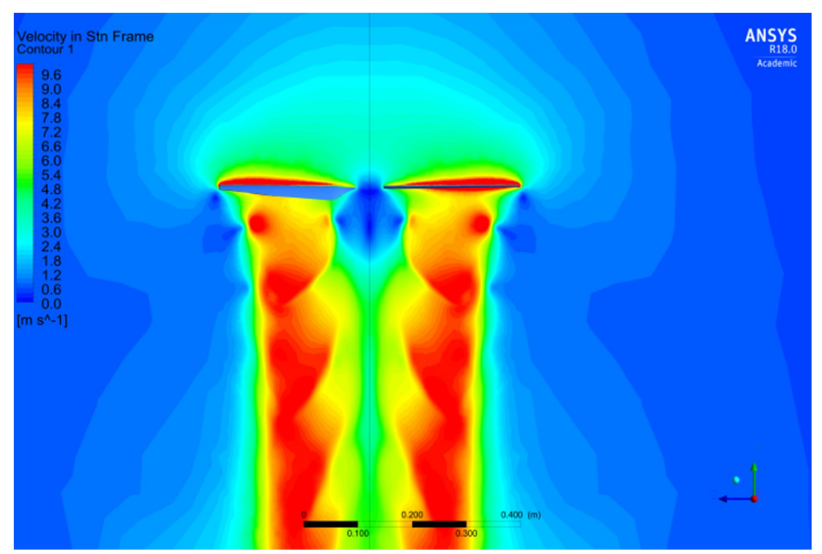

Fig. 6. Velocity map for $\mathrm{x}$-axis plane view.

Fig. 7 shows the close-up view on wing tip turbulence visualised by q-criterion iso-contour surface coloured by turbulence kinetic energy value. The profile turbulence transition region is visible.
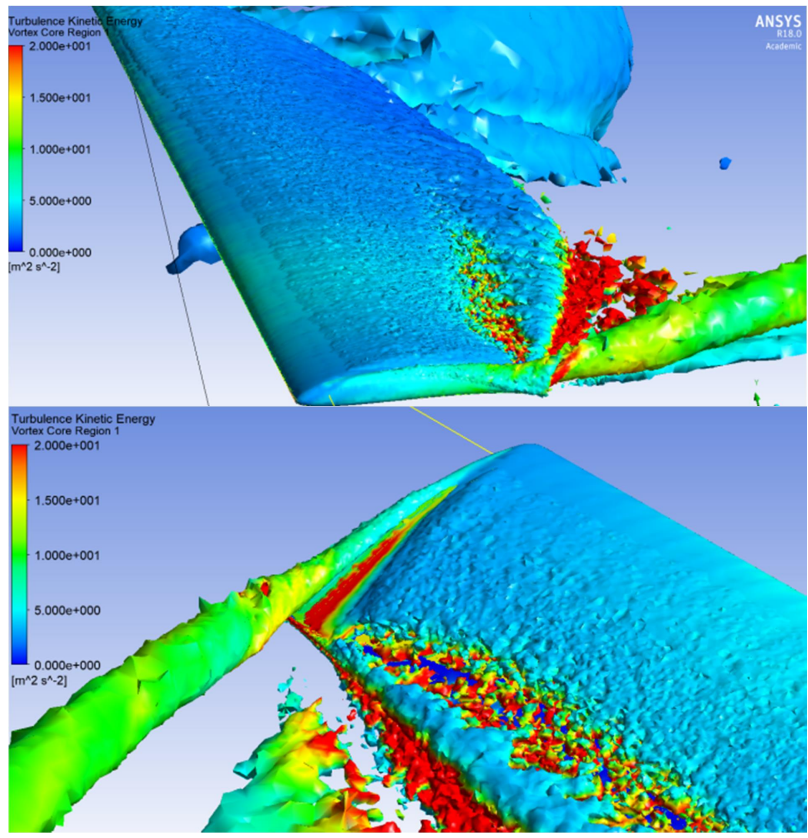

Fig. 7. Q-criterion iso-contour with turbulence kinetic energy color map -blade tip views.

The turbulence vortex sheet can be visualised by the vorticity iso-contour (Fig. 8).

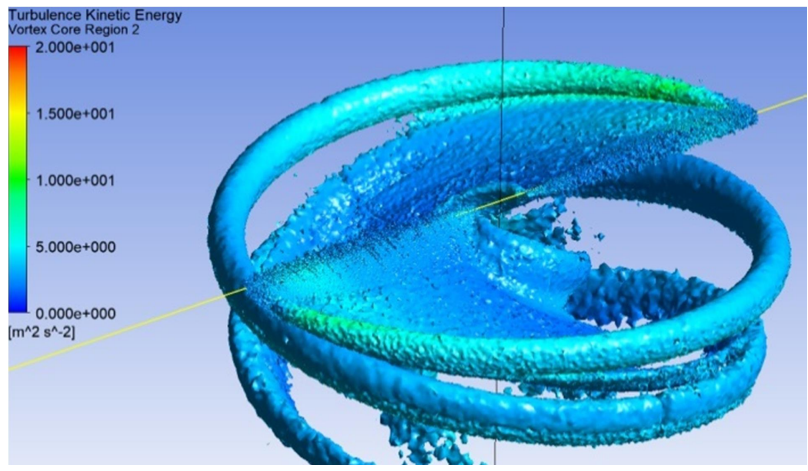

Fig. 8. Vorticity iso-contour with turbulence kinetic energy colour map.

\section{Conclusions}

Results prove the feasibility of low Reynolds Number at low Disk Loading. The results from theoretical model, BET analysis and CFD analysis prove that using low RE rotor blades is achievable. Such blades are beneficial in terms of lower energy usage, thus higher flight enduranceAt Disk Loading $5.76 \mathrm{~kg} / \mathrm{m} 2$ the inverse power loading value of $68 \mathrm{~W} / \mathrm{kg}$ was achieved allowing for around 50 minute hover endurance assuming that $50 \%$ of rotorcraft weight is a high density Li-ion battery. The results prove that low RE rotors can be beneficial in terms of lower energy use. Further research will investigate even lower values of disk loading and also the control capabilities of slowed rotor operating in low RE regime. The paper also proves that ANSYS software 
can be used to efficiently model a complicated flow through the rotor using the Frozen Rotor approach.

\section{References}

1. T. Tulwin, M. Klimek, P. Piech, ITM Web Conf. 15, 07010 (2017) DOI: 10.1051/itmconf/20171507010

2. J. C. Ho, H. Yeo, J. Fluids \& Structures 68, 194-223 (2017) DOI: 10.1016/j.jfluidstructs.2016.09.007

3. S. Sunada, C. P. Ellington, AIAA J 38,8, 13131321 (2000) DOI: $10.2514 / 2.1124$

4. M. R. A. Nabawy, W. J. Crowther, J. Royal Society Interface, 11, 93 (2014) DOI: 10.1098/rsif.2013.1196

5. M. Benedict, J. Winslow, Z. Hasnain, I. Chopra, Int. J. Micro Air Vehicles 7, 3, 231-255 (2015) DOI: 10.1260/1756-8293.7.3.231

6. J. Watkinson, The Art of The Helicopter, 1, (2004)
7. H. Otsuka, K. Nagatani, 34th AIAA Applied Aerodynamics Conference, (Am. Inst. Aeronautics \& Astronautics, 2016) DOI: 10.2514/6.2016-3423

8. J. M. Winslow, UMD Theses and Dissertations, 162 (2016)

9. M. Drela, M. B. Giles, AIAA J. 25, 10, 1347-1355 (1987) DOI: $10.2514 / 3.9789$

10. S. R. Hall, K. Y. Yang, K. C. Hall, J Aircraft 31, 4, 837-845 (1994) DOI: 10.2514/3.46569

11. R. Shrestha, M. Benedict, V. Hrishikeshavan, I. Chopra, J Aircraft 53, 4, 1160-1167 (2016) DOI: 10.2514/1.C033621

12. J. Köhler, J. Friedrich, A. Ostendorf, E. L. Gurevich, Phys. Rev. 93, 2 (2016), DOI: 10.1103/PhysRevE.93.023108

13. O. Eboibi, L. A. M. Danao, R. J. Howell, Renewable Energy 92, 474-483 (2016) DOI: 10.1016/j.renene.2016.02.028

14. M. K. Rwigema, 27, ICAS (2010) 